\title{
An Algorithm for Computing Cocyclic Matrices Developed over Some Semidirect Products
}

\author{
V. Álvarez, J.A. Armario, M.D. Frau, and P. Real ${ }^{\star}$ \\ Dpto. Matemática Aplicada I, Universidad de Sevilla, Avda. Reina Mercedes s/n \\ 41012 Sevilla, Spain, \\ \{valvarez, armario, mdfrau, real\}@us.es
}

\begin{abstract}
An algorithm for calculating a set of generators of representative 2-cocycles on semidirect product of finite abelian groups is constructed, in light of the theory over cocyclic matrices developed by Horadam and de Launey in [78]. The method involves some homological perturbation techniques [31, in the homological correspondent to the work which Grabmeier and Lambe described in 12 from the viewpoint of cohomology. Examples of explicit computations over all dihedral groups $D_{4 t}$ are given, with aid of MATHEMATiCA.
\end{abstract}

\section{Introduction}

Let $G$ be a group, $U$ a trivial $G$-module. Functions $\psi: G \times G \rightarrow U$ which satisfy $\psi(a, b) \psi(a b, c)=\psi(b, c) \psi(a, b c), a, b, c \in G$ are called 2-cocycles 19. A cocycle is a coboundary $\delta \alpha$ if it is derived from a set mapping $\alpha$ : $\rightarrow U$ having $\alpha(1)=1$ by $\delta \alpha(a, b)=\alpha(a)^{-1} \alpha(b)^{-1} \alpha(a b)$. For each $G$ and $U$, the set of cocycles forms an abelian group $Z^{2}(G, U)$ under pointwise multiplication, and the coboundaries form a subgroup $B^{2}(G, U)$. Two cocycles $\psi$ and $\psi^{\prime}$ are cohomologous if there exists a coboundary $\delta \alpha$ such that $\psi^{\prime}=\psi \cdot \delta \alpha$. Cohomology is an equivalence relation and the cohomology class of $\psi$ is denoted $[\psi]$. It follows that the quotient group $Z^{2}(G, U) / B^{2}(G, U)$ consisting of the cohomology classes, forms an abelian group $H^{2}(G, U)$, which is known as the second cohomology group of $G$ with coefficients in $U$. For each $n \geq 0$ one may define the cocycle analogous in dimension $n$ ( $n$-cocycle). In spite of the important role played by cocycles in Algebraic Topology, Representation Theory and Quantum Systems, the problem of explicitly determining a full representative set of $n$-cocycles for given $G$ and $U$ does not appear to have been traditionally studied by cohomologists, at least, till the last decade.

A 2-cocycle $\psi$ is naturally displayed as a cocyclic matrix (associated to $\psi$, developed over $G$ ); that is, a $|G| \times|G|$ square matrix whose rows and columns are indexed by the elements of $G$ (under some fixed ordering) and whose entry

\footnotetext{
* All authors are partially supported by the PAICYT research project FQM-296 from Junta de Andalucía and the DGESIC research project PB98-1621-C02-02 from Education and Science Ministry (Spain).
} 
in position $(g, h)$ is $\psi(g, h)$. This notion was fruitfully used by Horadam and de Launey 6715 proving some interesting connections between combinatorial design theory and 2-cocycles, as well as connections between coding theory and 2-cocycles. It is also apparent that cocyclic matrices, associated with cocycles with coefficients in $\mathbb{K}_{2}=\{-1,1\}$, account for large classes of so-called Hadamard matrices [8], and may consequently provide an uniform approach to the famous Hadamard conjecture.

These facts have yield that over the past decade considerable effort has been devoted to computations of cocycles and cocyclic matrices. Using classical methods involving the Universal Coefficient Theorem, Schur multipliers, inflation and transgression, two algorithms for finding 2-cocycles representing 2-dimensional cohomology classes can be worked out. The first one [78] applies to an abelian group $G$ and the second [10] over groups $G$ for which the word problem is solvable.

Horadam and de Launey's method is based on an explicit version of the wellknown Universal Coefficient Theorem, which provides a decomposition of the second cohomology group into the direct sum of two summands,

$$
H^{2}(G, U) \cong \operatorname{Ext}(G /[G, G], U) \oplus \operatorname{Hom}\left(H_{2}(G), U\right)
$$

These connections make possible the translation of cocyclic development onto a (co)homological framework.

This link becomes stronger noting the "Bar construction" 19] related to G. It is a DG-module, which consists of the $\mathbb{Z}$-modules

$$
M_{0}(G)=\mathbb{Z}, \quad M_{m}(G)=<\left[g_{1}, \ldots, g_{m}\right]: g_{i} \in G, 1 \leq i \leq m>
$$

and differential $\partial$,

$$
\begin{gathered}
\partial_{1}\left(\left[g_{1}\right]\right)=0, \quad \partial_{m+1}\left(\left[g_{1}, \ldots, g_{m+1}\right]\right)=(-1)^{m+1}\left(\left[g_{1}, \ldots g_{m}\right]\right)+ \\
+\left(\left[g_{2}, \ldots, g_{m+1}\right]\right)+\sum_{i=1}^{m}(-1)^{i}\left(\left[g_{1}, \ldots, g_{i} g_{i+1}, \ldots, g_{m+1}\right]\right) .
\end{gathered}
$$

The quotient $\operatorname{Ker}\left(\partial_{m}\right) / \operatorname{Im}\left(\partial_{m+1}\right)$ is known to be the $m^{\text {th }}$ integral homology group of $G, H_{m}(G)$. Let $R_{2}(G)$ denote the quotient $M_{2}(G) / \operatorname{Im}\left(\partial_{3}\right) \supseteq H_{2}(G)$.

Taking into account what $\partial_{3}$ means, it is readily checked that the map

$$
\begin{array}{cl}
\phi: Z^{2}\left(G, \mathbb{K}_{2}\right) & \rightarrow \operatorname{Hom}\left(R_{2}(G), \mathbb{K}_{2}\right) \\
h & \mapsto \quad \phi(h)
\end{array}
$$

such that

$$
\phi(h)\left(\sum_{(a, b) \in G \times G} \lambda_{(a, b)}(a, b)+\operatorname{Im}\left(\partial_{2}\right)\right)=\sum_{(a, b) \in G \times G} \lambda_{(a, b)} h[a, b]
$$

defines an isomorphism between the set of 2-cocycles and $\operatorname{Hom}\left(R_{2}(G), \mathbb{K}_{2}\right)$ [7]. 
The problem of computing a set of generators for 2-cocycles hence translates to the problem of determining a set of coboundary, symmetric and commutator generators, such that

$$
Z^{2}\left(G, \mathbb{K}_{2}\right) \cong B^{2}\left(G, \mathbb{K}_{2}\right) \oplus \operatorname{Ext}_{\mathbb{Z}}\left(G /[G, G], \mathbb{K}_{2}\right) \oplus \operatorname{Hom}\left(H_{2}(G), \mathbb{K}_{2}\right) .
$$

A minimal set for symmetric generators may be calculated from a primary invariant decomposition of $G /[G, G] \cong H_{1}(G)$, as a Kronecker product of back negacyclic matrices [7]. A minimal set for coboundary generators is derived from the multiplication table of $G$ by means of linear algebra manipulations. But it is far from clear how to get a minimal set for commutator generators, in general. One should try to compute the second homology group of $G$ by means of $\left(M_{2}, \partial_{2}, \partial_{3}\right)$. Indeed, $\partial_{2}$ is not needed for finite groups $G$, since $H_{2}(G)$ is a direct sum of finite cyclic groups as it is the case. This procedure is not suitable in practice, since matrices involved are large in most cases.

On the other hand, Flannery calculates these summands as the images of certain embeddings which are complementary, called inflation and transgression. Calculation of representative 2-cocycles associated to $\operatorname{Ext}(G /[G, G], U)$ (inflation) is canonical. However, calculation of a complement of the image by the embeddings of inflation in $H^{2}(G, U)$ as the image of transgression is not canonical, anyway. As a matter of fact, it depends on the choice of a Schur complement. This is a potential source of difficulties in computation of representative 2-cocycles associated with elements of $\operatorname{Hom}\left(H_{2}(G), U\right)$. This method has already been implemented in [11, using the symbolic computational system MAGMA.

Using a far different approach, Grabmeier and Lambe present in [12] alternate methods for calculating representative 2-cocycles for all finite $p$-groups from the point of view of Homological Perturbation Theory [13 1420]. The computer algebra system Ахіом has been used in order to make calculations in practice.

Here we present a method for explicitly determining a full set of representative 2-cocycles for the elements of the second cohomology group $H^{2}(G, \mathbb{Z})$ where $G$ is $\mathbb{Z}_{r} \times_{\chi} \mathbb{Z}_{s}$. All general statements given in this paper are applicable to any semidirect product of finite abelian groups, but for simplicity in the exposition, for this class, only the case $\mathbb{Z}_{r} \times_{\chi} \mathbb{Z}_{s}$ will be presented.

Our method could be seen as a mixture of both the algorithms given by Flannery in 10] and Grabmeier-Lambe in [12. Indeed, we compute representative 2-cocycles proceeding from $\operatorname{Hom}\left(H_{2}\left(\mathbb{Z}_{r} \times_{\chi} \mathbb{Z}_{s}\right), \mathbb{K}_{2}\right)$. This alternate method is based on some Homological Perturbation techniques developed in the work of authors [3,1] on the determination of "homological models" (those differential graded modules $h G$ with $H_{n}(G)=H_{n}(h G)$, see [4 for instance), for semidirect products of finite abelian groups with group action. The algorithm is straightforward enough to be programmed in any computer algebra system, as we have done in Mathematica[2].

The main steps are to define functions and $F: M_{2}\left(\mathbb{Z}_{r} \times_{\chi} \mathbb{Z}_{s}\right) \rightarrow V_{2}$ and $d_{i}: V_{i} \rightarrow V_{i-1}$, where $V_{i}$ are certain "perturbed" simple algebras. These will be defined in such a way that for any representative 2-cycle $z$ in the quotient ker $d_{2} / \operatorname{Im} d_{3}$, the elevation of $z$ through $F$ will define a representative 2 -cocycle. This is the homology analogous to the work of Grabmeier-Lambe in [12]. 
It should be noted that explicit formulae for representative 2-cocycles of $H^{2}\left(\mathbb{Z}_{r} \times_{\chi} \mathbb{Z}_{s}, \mathbb{Z}_{2}\right)$ are given in [21]. The approach explained in this paper covers the more general case of any semidirect product of finite abelian groups.

Similar algorithms may be considered to reach many other settings, progressing from any finite group with known homological model.

\section{The Algorithm}

Let $\mathbb{Z}_{r} \times_{\chi} \mathbb{Z}_{s}$ be a semidirect product, $\chi$ a group action such that

$$
\left(a_{1}, b_{1}\right) \cdot\left(a_{2}, b_{2}\right)=\left(a_{1}+\chi\left(b_{1}, a_{2}\right), b_{1}+b_{2}\right), \quad a_{1}, a_{2} \in \mathbb{Z}_{r}, b_{1}, b_{2} \in \mathbb{Z}_{s} .
$$

Let consider the following auxiliary sets

$$
\begin{gathered}
V_{2}=\mathbb{Z}\left[x^{2}, x y, y^{2}\right], \quad V_{3}=\mathbb{Z}\left[x^{3}, x^{2} y, x y^{2}, y^{3}\right], \\
B_{2}=\{[n, m] \otimes[]: 1 \leq n, m<r\} \cup\{[n] \otimes[m]: 1 \leq n<r, 1 \leq m<s\} \cup \\
\cup\{[] \otimes[n, m]: 1 \leq n, m<s\},
\end{gathered}
$$

$B_{3}=\{[n, m, k] \otimes[]: 1 \leq n, m, k<r\} \cup\{[n, m] \otimes[k]: 1 \leq n, m<r, 1 \leq k<s\} \cup$

$\cup\{[n] \otimes[m, k]: 1 \leq n<r, 1 \leq m, k<s\} \cup\{[] \otimes[n, m, k]: 1 \leq n, m, k<s\}$.

We will define $\mathbb{Z}$-linear functions $g_{3}: V_{3} \rightarrow B_{3}, f_{i}: B_{i} \rightarrow V_{i}$ for $i=2,3$, $\phi_{2}: B_{2} \rightarrow B_{3}, \rho_{3}: B_{3} \rightarrow B_{2}, d_{3}: V_{3} \rightarrow V_{2}$ and $f_{\infty}: B_{2} \rightarrow V_{2}$. Let

$$
\begin{aligned}
g_{3}\left(x^{3}\right) & =([1,1,1]+\cdots+[1, r-1,1]) \otimes[], \\
g_{3}\left(x^{2} y\right) & =([1,1]+\cdots+[1, r-1]) \otimes[1], \\
g_{3}\left(x y^{2}\right) & =[1] \otimes([1,1]+\cdots+[1, s-1]), \\
g_{3}\left(y^{3}\right) & =[] \otimes([1,1,1]+\cdots+[1, r-1,1]) \\
f_{2}([n, m] \otimes[]) & =x^{2}, \text { if } n+m \geq r \\
f_{2}([n] \otimes[m]) & =(n m) x y \\
f_{2}([] \otimes[n, m]) & =y^{2}, \text { if } n+m \geq s, \\
f_{3}([n, m, k] \otimes[]) & =k x^{3}, \text { if } n+m \geq r, \\
f_{3}([n, m] \otimes[k]) & =k x^{2} y, \text { if } n+m \geq r \\
f_{3}([n] \otimes[m, k]) & =n x y^{2}, \text { if } m+k \geq s, \\
f_{3}([] \otimes[n, m, k]) & =k y^{3}, \text { if } n+m \geq s, \\
\left.\phi_{2}([n, m] \otimes[]]\right) & =-([1,1, m]+\cdots[1, n-1, m]) \otimes[] \\
\phi_{2}([n] \otimes[m]) & =-([1,1]+\cdots[1, n-1]) \otimes[m]+[n] \otimes([1,1]+\cdots[1, m-1]), \\
\phi_{2}([] \otimes[n, m]) & =-[] \otimes([1,1, m]+\cdots[1, n-1, m]) \\
\rho_{3}([n, m] \otimes[k]) & =[\chi(k, n), \chi(k, m)] \otimes[]-[n, m] \otimes[], \\
\rho_{3}([n] \otimes[m, k]) & =[n] \otimes[k]-[\chi(m, n)] \otimes[k], \\
D_{3}\left(x^{2} y\right) & =r x y, \\
D_{3}\left(x y^{2}\right) & =-s x y .
\end{aligned}
$$


These morphisms are understood to be zero otherwise. Let define

$$
d_{3}=D_{3}+f_{2} \rho_{3} g_{3}-f_{2} \rho_{3} \phi_{2} \rho_{3} g_{3}+f_{2}\left(\rho_{3} \phi_{2}\right)^{2} \rho_{3} g_{3}-\cdots,
$$

and $f_{\infty}: B_{2} \rightarrow V_{2}$,

$$
f_{\infty}=f_{2}-f_{2} \rho_{3} \phi_{2}+f_{2}\left(\rho_{3} \phi_{2}\right)^{2}-\cdots
$$

Geometric series of these types converge to define a map, as it is proved in the more general setting of generalized semidirect products of finite abelian groups in $\left[1\right.$. The fact is that $\rho_{*}$ decreases the dimension on the second component, and $\phi_{*}$ either increments the dimension only on the first component or decreases the value of the element in the second component. Hence the composition $\phi_{i-1} \rho_{i}$ becomes nilpotent.

Notice that the sets $B_{i}$ defined above consist of the products

$$
B_{i}=\bigoplus_{0 \leq j \leq i}\left(M_{j}\left(\mathbb{Z}_{r}\right) \otimes M_{i-j}\left(\mathbb{Z}_{s}\right)\right) .
$$

There is a connecting map $F_{2}: M_{2}\left(\mathbb{Z}_{r} \times_{\chi} \mathbb{Z}_{s}\right) \rightarrow B_{2}$, so that

$$
\begin{gathered}
F_{2}\left[\left(a_{1}, b_{1}\right),\left(a_{2}, b_{2}\right)\right]=[] \otimes\left[b_{2}, b_{1}\right]+2\left[\chi\left(b_{2}, a_{2}\right)\right] \otimes\left[b_{1}\right]+2\left[\chi\left(b_{2}, a_{2}\right), \chi\left(b_{2} b_{1}, a_{1}\right)\right] \otimes[]- \\
-\left[\chi\left(b_{2} b_{1} b_{2}, a_{2}\right), \chi\left(b_{2} b_{1} b_{2} b_{1}, a_{1}\right)\right] \otimes[]-\left[\chi\left(b_{2} b_{2}, a_{2}\right)\right] \otimes\left[b_{1}\right] .
\end{gathered}
$$

Theorem 1. Assume the notation above.

1. $H_{2}\left(\mathbb{Z}_{r} \times_{\chi} \mathbb{Z}_{s}\right)=H_{2}\left(V_{2}\right)$, which is computed from $d_{3}$.

2. The map $F=f_{\infty} \circ F_{2}: M_{2}\left(\mathbb{Z}_{r} \times_{\chi} \mathbb{Z}_{s}\right) \rightarrow V_{2}$ induces an isomorphism in homology, such that for any $z \in H_{2}\left(V_{2}\right)$ the elevation of $z$ through $F$ defines a cocyclic matrix over $\mathbb{Z}_{r} \times_{\chi} \mathbb{Z}_{s}$.

In 1 the authors find a homological model for semidirect products of finite abelian groups. In particular, attending to the groups $\mathbb{Z}_{r} \times_{\chi} \mathbb{Z}_{s}$, it is proved that $H_{2}\left(M_{2}\left(\mathbb{Z}_{r} \times \mathbb{Z}_{s}\right)=H_{2}\left(V_{2}\right)\right.$. Moreover $F$ is shown to induce an isomorphism in homology.

Nevertheless the formula for $F$ is not explicitly given there, since it is complicated to give an explicit formula for $f_{\infty}$ in the general case of semidirect products of groups.

It is a remarkable fact that for every finite group $G, H_{2}(G)$ is a finite abelian group [5]. This way, it is only needed $d_{3}$ in order to compute $H_{2}\left(V_{2}\right)$ by means of Veblen's algorithm [22.

This process consists in calculating the integer Smith normal form $D$ of the matrix $M$ representing $d_{3}$ with regards to basis $\mathcal{B}=\left\{x^{3}, x^{2} y, x y, y^{3}\right\}$ and $\mathcal{B}^{\prime}=\left\{x^{2}, x y, y^{2}\right\}$.

Let $\mathcal{U}=\left\{u_{1}, u_{2}, u_{3}, u_{4}\right\}$ and $\mathcal{V}=\left\{v_{1}, v_{2}, v_{3}\right\}$ define these change basis, such that $D_{\mathcal{U}, \mathcal{V}}=P M_{\mathcal{B}, \mathcal{B}^{\prime}} Q$, for appropriated change basis matrices $P$ and $Q$.

Now we explain what we mean with "elevate $z$ through $F$ ". 
We want to determine all cocyclic matrices over $\mathbb{Z}_{r} \times_{\chi} \mathbb{Z}_{s}$. That is, all representative 2 -cocycles of $\mathbb{Z}_{r} \times_{\chi} \mathbb{Z}_{s}$. Thus it suffices to calculate which $x$ in $M_{2}\left(\mathbb{Z}_{r} \times_{\chi} \mathbb{Z}_{s}\right)$ are shown to give non trivial homological information in $H_{2}\left(V_{2}\right)$.

For each generator $z$ in $H_{2}\left(V_{2}\right)$, the elevation of $z$ through $F$ relates to the set of elements in $M_{2}\left(\mathbb{Z}_{r} \times{ }_{\chi} \mathbb{Z}_{s}\right)$ which projects onto $z$ with 2-homological information. This can be achieved in two single elevations: one from $V_{2}$ to $B_{2}$, the other from $B_{2}$ to $M_{2}\left(\mathbb{Z}_{r} \times_{\chi} \mathbb{Z}_{s}\right)$.

¿From the theorem above, an algorithm for calculating representative 2cocycles may be derived in a straightforward manner.

Notice that map $F$ should be called the universal 2-cochain, following Grabmeier-Lambe's notation in [12].

Algorithm 1 InPUT DATA: a semidirect product $\mathbb{Z}_{r} \times_{\chi} \mathbb{Z}_{s}$.

Step 1. Compute $d_{3}: V_{3} \rightarrow V_{2}$, the differential of the homological model of $\mathbb{Z}_{r} \times_{\chi} \mathbb{Z}_{s}$ in dimension 3 .

Step 2. Compute $H_{2}\left(\mathbb{Z}_{r} \times_{\chi} \mathbb{Z}_{s}\right)$ and representative cycles from $d_{3}$.

Step 3. Elevate the representative cycles from $H_{2}\left(\mathbb{Z}_{r} \times_{\chi} \mathbb{Z}_{s}\right)$ to $M_{2}\left(\mathbb{Z}_{r} \times_{\chi} \mathbb{Z}_{s}\right)$ via $F$.

OutPut Data: Set of commutator generators for a basis of cocyclic matrices over $\mathbb{Z}_{r} \times \mathbb{Z}_{s}$.

It should be taken into account that Step $\mathbf{2}$ often requires to compute the Smith normal form of the matrix corresponding to $d_{3}$, which is always of size $4 \times 3$, independently of indexes $r$ and $s$ of the factors. This is the fundamental improvement in the calculus of the commutator generators, since the size of matrices which arises from the complex $\left(M_{*}, \partial_{*}\right)$ depends on the order of the group (the matrix corresponding to operator $\partial_{3}$ is of size $(r s)^{3} \times(r s)^{2}$ for the semidirect product $\mathbb{Z}_{r} \times_{\chi} \mathbb{Z}_{s}$ ).

It may be possible to extend the Theorem 1 and its associated algorithm to other certain families of groups, with homological models already known, such as central extensions [18, finitely generated torsion free nilpotent groups [16], metacyclic groups [17] and many others. It is only needed to find explicit formulae for the analogous of maps $F_{2}$ and $F$.

\section{An Example: Dihedral Groups $D_{2 t \cdot 2}$}

In this section we apply Algorithm 1 in the particular case of dihedral groups. A Mathematica program is used, which authors provide in [2].

It should be noted that dihedral groups $D_{t .2}$ for odd values of $t$ do not provide 2-homological information, since $H_{2}\left(D_{t \cdot 2}\right)$ is known to be zero in this case.

Let $D_{2 t \cdot 2}=\{(0,0),(1,0), \ldots,(2 t-1,0),(1,1), \ldots,(2 t-1,1)\}$,

$$
\chi(0, n)=n, \quad \chi(1, n)=2 t-n, \quad \forall n \in \mathbb{Z}_{2 t} .
$$


An explicit formula for $F$ can be worked out for these groups, so that if we define $\lambda: \mathbb{Z}_{k} \times \mathbb{Z}_{k} \rightarrow \mathbb{Z}_{2}, k \geq 2$, as $\lambda[x, y]=1$ if $x+y \geq k$ and 0 otherwise, it is readily checked that

$$
\begin{gathered}
F\left[\left(a_{1}, b_{1}\right),\left(a_{2}, b_{2}\right)\right]=b_{1} b_{2} y^{2}+2 b_{1} \chi\left(b_{2}, a_{2}\right) x y+2 b_{1}\left(\chi\left(b_{2}, a_{2}\right)-1\right) x^{2}+ \\
+2 \lambda\left[\chi\left(b_{2}, a_{2}\right), \chi\left(b_{2} b_{1}, a_{1}\right)\right] x^{2}-\lambda\left[\chi\left(b_{1}, a_{2}\right), a_{1}\right] x^{2}-a_{2} b_{1} x y-b_{1}\left(a_{2}-1\right) x^{2} .
\end{gathered}
$$

Let consider the cases $t=1, D_{2 \cdot 2}=\{(0,0),(1,0),(0,1),(1,1)\}$,

$t=2, D_{4 \cdot 2}=\{(0,0),(1,0),(2,0),(3,0),(0,1),(1,1),(2,1),(3,1)\}$, and $t=6, D_{12 \cdot 2}=\{(0,0),(1,0), \ldots,(11,0),(0,1),(1,1), \ldots,(11,1)\}$.

Step 1. Compute $d_{3}$.

\begin{tabular}{|c|c|c|c|}
\hline$d\left(V_{3}\right)$ & $t=1$ & $t=2$ & $t=6$ \\
\hline$x^{3}$ & 0 & 0 & 0 \\
\hline$x^{2} y$ & $2 x y$ & $2 x^{2}+4 x y$ & $10 x^{2}+12 x y$ \\
\hline$x y^{2}$ & $-2 x y$ & $-2 x^{2}-4 x y$ & $-10 x^{2}-12 x y$ \\
\hline$y^{3}$ & 0 & 0 & 0 \\
\hline
\end{tabular}

Step 2. Compute $H_{2}\left(D_{2 t \cdot 2}\right)$ and representative cycles from $d_{3}$.

In order to compute $H_{2}\left(D_{2 t \cdot 2}\right)$ in the cases $t=1,2,6$, it is useful to calculate the Smith normal form $D_{t}=P_{t} M_{t} Q_{t}$ of the matrix $M_{t}$ associated to $d_{3}$, with basis change matrices $P_{t}$ and $Q_{t}$, respectively. In these cases,

\begin{tabular}{|c|c|c|c|}
\hline & $t=1$ & $t=2$ & $t=6$ \\
\hline$D_{t}$ & $\left(\begin{array}{lll}2 & 0 & 0 \\
0 & 0 & 0 \\
0 & 0 & 0 \\
0 & 0 & 0\end{array}\right)$ & $\left(\begin{array}{lll}2 & 0 & 0 \\
0 & 0 & 0 \\
0 & 0 & 0 \\
0 & 0 & 0\end{array}\right)$ & $\left(\begin{array}{lll}2 & 0 & 0 \\
0 & 0 & 0 \\
0 & 0 & 0 \\
0 & 0 & 0\end{array}\right)$ \\
\hline$Q_{t}$ & $\left(\begin{array}{lll}0 & 1 & 0 \\
1 & 0 & 0 \\
0 & 0 & 1\end{array}\right)$ & $\left(\begin{array}{rrr}1 & -2 & 0 \\
0 & 1 & 0 \\
0 & 0 & 1\end{array}\right)$ & $\left(\begin{array}{rrr}-1 & -6 & 0 \\
1 & 5 & 0 \\
0 & 0 & 1\end{array}\right)$ \\
\hline
\end{tabular}

Hence, $H_{2}\left(D_{2 t \cdot 2}\right)=\mathbb{Z}_{2}$ for $t=1,2,6$ and the representative cycle is the first element in the new basis $\mathcal{U}$ of $\mathbb{Z}\left[V_{2}\right]$.

In order to translate to the basis $\mathcal{B}$ of $\mathbb{Z}\left[V_{2}\right]$ the homological information which $H_{2}\left(D_{2 t \cdot 2}\right)$ provides, it suffices to select the odd entries of each of the columns of $Q_{t}$ corresponding to each representative cycle in the basis $\mathcal{U}$ (that is, to select which elements of $\mathbb{Z}\left[V_{2}\right]$ with regards to basis $\mathcal{B}$ have an odd entry in the position corresponding to a representative cycle with coordinates in basis $\mathcal{U}$ ). The homological information is concentrated in elements with coordinates $(-, n,-)_{\mathcal{B}}$ for odd values of $n$ in the case $t=1$, in elements $(n,-,-)_{\mathcal{B}}$ for odd values of $n$ in the case $t=2$, and in elements $(n, m,-)_{\mathcal{B}}$ for $n, m$ of distinct parity in the case $t=6$. 
Step 3. Elevate the representative cycles from $H_{2}\left(D_{2 t \cdot 2}\right)$ to $M_{2}\left(D_{2 t \cdot 2}\right)$ via $F$. It suffices to detect which elements of $M_{2}\left(D_{2 t \cdot 2}\right)$ are carried out via $F$ to elements $(-, n,-)_{\mathcal{B}}$ for odd $n(t=1),(n,-,-)_{\mathcal{B}}$ for odd $n(t=2)$, and $(n, m,-)_{\mathcal{B}}$ for $n, m$ of distinct parity $(t=6)$. These elements indicate the positions in the $\left|D_{2 t \cdot 2}\right| \times\left|D_{2 t \cdot 2}\right|$ commutator cocyclic generator matrix which are not trivial.

In the case $t=1$, we obtain the following elements:

$$
[(0,1),(1,0)],[(1,1),(1,0)],[(0,1),(1,1)],[(1,1),(1,1)] .
$$

For $t=2$,

$[(1,0),(3,0)],[(1,0),(3,1)],[(2,0),(2,0)],[(2,0),(3,0)],[(2,0),(2,1)]$,

$[(2,0),(3,1)],[(3,0),(1,0)],[(3,0),(2,0)],[(3,0),(3,0)],[(3,0),(0,1)]$,

$[(3,0),(2,1)],[(3,0),(3,1)],[(0,1),(2,0)],[(0,1),(2,1)],[(1,1),(1,0)]$,

$[(1,1),(2,0)],[(1,1),(1,1)],[(1,1),(3,1)],[(2,1),(1,0)],[(2,1),(1,1)]$,

$[(3,1),(1,0)],[(3,1),(3,0)],[(3,1),(1,1)],[(3,1),(3,1)]$.

In the case $t=6$, the elements which are carried out via $F$ to elements $(n, m,-)_{\mathcal{B}}$ for $n, m$ of distinct parity are those $\left[\left(a_{1}, b_{1}\right),\left(a_{2}, b_{2}\right)\right]$ such that

$$
\left\{\begin{array}{l}
b_{1}=0, a_{1}+a_{2}>11 \\
\text { or } \\
b_{1}=1, a_{1}<a_{2} .
\end{array}\right.
$$

Output data: set of commutator generators for a basis of cocyclic matrices over $D_{2 t \cdot 2}, t=1,2,6$. Assuming $K^{2}=1$, we obtain

\begin{tabular}{|c|c|c|}
\hline$t=1$ & $t=2$ & $t=6$ \\
\hline$\left(\begin{array}{ll}A_{1} & A_{1} \\
B_{1} & B_{1}\end{array}\right)$ & $\left(\begin{array}{ll}A_{2} & A_{2} \\
B_{2} & B_{2}\end{array}\right)$ & $\left(\begin{array}{ll}A_{6} & A_{6} \\
B_{6} & B_{6}\end{array}\right)$ \\
\hline
\end{tabular}

where

$$
\begin{gathered}
A_{1}=\left(\begin{array}{ll}
1 & 1 \\
1 & 1
\end{array}\right), \quad B_{1}=\left(\begin{array}{ll}
1 & K \\
1 & K
\end{array}\right), \quad A_{2}=\left(\begin{array}{cccc}
1 & 1 & 1 & 1 \\
1 & 1 & 1 & K \\
1 & 1 & K & K \\
1 & K & K & K
\end{array}\right), \quad B_{2}=\left(\begin{array}{cccc}
1 & 1 & K & 1 \\
1 & K & K & 1 \\
1 & K & 1 & 1 \\
1 & K & 1 & K
\end{array}\right), \\
A_{6}=\left(\begin{array}{ccccc}
1 & 1 & \cdots & 1 & 1 \\
1 & 1 & \cdots & 1 & K \\
\vdots & \vdots & & \vdots & \vdots \\
1 & 1 & \cdots & K & K \\
1 & K & \cdots & K & K
\end{array}\right), \quad B_{6}=\left(\begin{array}{ccccc}
1 & K & \cdots & K & K \\
1 & 1 & \cdots & K & K \\
\vdots & \vdots & & \vdots & \vdots \\
1 & 1 & \cdots & 1 & K \\
1 & 1 & \cdots & 1 & 1
\end{array}\right) .
\end{gathered}
$$

Note that $A_{6}$ is usually called back negacyclic.

In general, it may be proved that for $t>2$ the computation of $H_{2}\left(D_{2 t \cdot 2}\right)$ reduces to the matrices

$$
D_{t}=\left(\begin{array}{lll}
2 & 0 & 0 \\
0 & 0 & 0 \\
0 & 0 & 0 \\
0 & 0 & 0
\end{array}\right) \quad \text { and } \quad Q_{t}=\left(\begin{array}{ccc}
-1 & -t & 0 \\
1 & t-1 & 0 \\
0 & 0 & 1
\end{array}\right)
$$


so that $H_{2}\left(D_{2 t \cdot 2}\right)=\mathbb{Z}_{2}$ and the homological information is concentrated in elements with coordinates $(n, m,-)_{\mathcal{B}}$ for $n, m$ of distinct parity.

Hence, the set of commutator generators for a basis of cocyclic matrices over $D_{2 t \cdot 2}$ reduces to $\left\{\left(\begin{array}{c}A_{t} \\ A_{t} \\ B_{t} B_{t}\end{array}\right)\right\}$, where $A_{t}$ is the correspondant back negacyclic matrix and $B_{t}$ consists in the matrix whose rows are the ones of $A_{t}$ displayed in reverse order.

It should be noted that the cocyclic matrices over dihedral groups have already been found from Flannery's techniques in 10.

Remark 1. The case $t=2$ is also studied in [7, where the commutator generator is said to be

$$
\left(\begin{array}{llllllll}
1 & 1 & 1 & 1 & 1 & 1 & 1 & 1 \\
1 & 1 & 1 & B & B & 1 & 1 & 1 \\
1 & 1 & B & B & B & 1 & 1 & B \\
1 & B & B & B & B & 1 & B & B \\
1 & 1 & 1 & 1 & 1 & 1 & 1 & 1 \\
1 & B & B & B & B & 1 & B & B \\
1 & 1 & B & B & B & 1 & 1 & B \\
1 & 1 & 1 & B & B & 1 & 1 & 1
\end{array}\right)
$$

with $B^{2}=1$.

Both matrices differ in the (Hadamard) product of a coboundary generator $C$ and a symmetric generator $S$, which are

$$
C=\left(\begin{array}{llllllll}
1 & 1 & 1 & 1 & 1 & 1 & 1 & 1 \\
1 & A & 1 & A & A & A & 1 & 1 \\
1 & 1 & 1 & 1 & A & 1 & A & 1 \\
1 & A & 1 & A & A & 1 & 1 & A \\
1 & 1 & A & 1 & 1 & 1 & A & 1 \\
1 & A & 1 & 1 & A & 1 & 1 & 1 \\
1 & A & A & A & A & A & 1 & A \\
1 & 1 & 1 & A & A & 1 & 1 & 1
\end{array}\right), \quad S=\left(\begin{array}{llllllll}
1 & 1 & 1 & 1 & 1 & 1 & 1 & 1 \\
1 & D & 1 & D & 1 & D & 1 & D \\
1 & 1 & 1 & 1 & 1 & 1 & 1 & 1 \\
1 & D & 1 & D & 1 & D & 1 & D \\
1 & 1 & 1 & 1 & 1 & 1 & 1 & 1 \\
1 & D & 1 & D & 1 & D & 1 & D \\
1 & 1 & 1 & 1 & 1 & 1 & 1 & 1 \\
1 & D & 1 & D & 1 & D & 1 & D
\end{array}\right)
$$

with $A=D=-1$.

The matrix $C$ arises from any of the set map $\alpha_{k}: D_{4 \cdot 2} \rightarrow \mathbb{K}_{2}, k \in\{1,-1\}$,

$$
\begin{gathered}
\alpha(0,0)=1, \quad \alpha(1,0)=-1, \quad \alpha(2,0)=-1, \quad \alpha(3,0)=1, \\
\alpha(0,1)=k, \quad \alpha(1,1)=k, \quad \alpha(2,1)=k, \quad \alpha(3,1)=-k .
\end{gathered}
$$

\section{References}

1. V. Álvarez, J.A. Armario, M.D. Frau and P. Real. Homology of semidirect products of finite abelian groups with group action. Preprint of Dpto. Matemática Aplicada I, University of Seville (Spain, 2001). 
2. V. Álvarez, J.A. Armario, M.D. Frau and P. Real. Homology of semidirect products of groups: algorithms and applications. Preprint of Dpto. Matemática Aplicada I, University of Seville (Spain, 2001).

3. V. Álvarez, J.A. Armario, P. Real. On the homology of semi-direct products of groups. Colloquium on Topology, Gyula (Hungary, 1998).

4. J.A. Armario, P. Real and B. Silva. On $p$-minimal homological models of twisted tensor products of elementary complexes localized over a prime. Contemporary Mathematics, 227, 303-314, (1999).

5. K.S. Brown. Cohomology of groups. Graduate Texts in Math., 87, Springer-Verlag, New York (1982).

6. W. de Launey and K.J. Horadam. A weak difference set construction for higherdimensional designs. Designs, Codes and Cryptography, 3, 75-87, (1993).

7. W. de Launey and K.J. Horadam. Cocyclic development of designs, J. Algebraic Combin., 2 (3), 267-290, 1993. Erratum: J. Algebraic Combin., (1), pp. 129, 1994.

8. W. de Launey and K.J. Horadam. Generation of cocyclic Hadamard matrices. Computational algebra and number theory (Sydney, 1992), volume 325 of Math. Appl., 279-290. Kluwer Acad. Publ., Dordrecht, (1995).

9. D.L. Flannery. Transgression and the calculation of cocyclic matrices. Australas. J. Combin., 11, 67-78, (1995).

10. D.L. Flannery. Calculation of cocyclic matrices. J. of Pure and Applied Algebra, 112, 181-190, (1996).

11. D.L. Flannery and E.A. O'Brien. Computing 2-cocycles for central extensions and relative difference sets. Comm. Algebra, 28(4), 1939-1955, (2000).

12. J. Grabmeier, L.A. Lambe. Computing Resolutions Over Finite $p$-Groups. Proceedings ALCOMA'99. Eds. A. Betten, A. Kohnert, R. Lave, A. Wassermann. Springer Lecture Notes in Computational Science and Engineering, Springer-Verlag, Heidelberg, (2000).

13. V.K.A.M. Gugenheim and L.A. Lambe. Perturbation theory in Differential Homological Algebra, I. Illinois J. Math., 33, 556-582, (1989).

14. V.K.A.M. Gugenheim, L.A. Lambe and J.D. Stasheff. Perturbation theory in Differential Homological Algebra II, Illinois J. Math., 35 (3), 357-373, (1991).

15. K.J. Horadam and A.A.I. Perera. Codes from cocycles. Lecture Notes in Computer Science, volume 1255, 151-163, Springer-Verlag, Berlin-Heidelberg-New York, (1997).

16. J. Huebschmann. Cohomology of nilpotent groups of class 2. J. Alg. , 126, 400-450, (1989).

17. J. Huebschmann. Cohomology of metacyclic groups. Transactions of the American Mathematical Society, 328 (1), 1-72, (1991).

18. L.A. Lambe and J.D. Stasheff. Applications of perturbation theory to iterated fibrations. Manuscripta Math., 58, 367-376, (1987).

19. S. Mac Lane. Homology. Classics in Mathematics Springer-Verlag, Berlin, (1995). Reprint of the 1975 edition.

20. P. Real. Homological Perturbation Theory and Associativity. Homology, Homotopy and Applications, 2, 51-88, (2000).

21. K. Tahara. On the Second Cohomology Groups of Semidirect Products. Math Z., 129, 365-379, (1972).

22. O. Veblen. Analisis situs. A.M.S. Publications, 5, (1931). 